\title{
Splenic Abscesses as a Complication of Urinary Tract Infection: Case Report and Literature Review
}

\author{
Jarjou'I $A^{* 1}$, Benaya $A^{2}$, Pavlov $\mathrm{N}^{2}$, Tabi $\mathrm{M}^{2}$ and Munter $\mathrm{G}^{1}$
}

${ }^{1}$ Department of Medicine C, Shaare Zedek Medical Center, affiliated with the Hadassah-Hebrew University School of Medicine, Jerusalem, Israel

${ }^{2}$ Division of Internal Medicine, Shaare Zedek Medical Center, affiliated with the Hadassah-Hebrew University School of Medicine, Jerusalem, Israel

*Corresponding author: Jarjou'I A, Department of Medicine C, Shaare Zedek Medical Center, P.O Box 3235, Jerusalem 91031, Israel, Fax: +972-2-6555354, Tel: +972-5-25299573, E-mail: amir_jarjoui@hotmail.com

Citation: Jarjou'I A, Benaya A, Pavlov N, Tabi M, Munter G (2019) Splenic Abscesses as a Complication of Urinary Tract Infection: Case Report and Literature Review. J Case Rep Stud 7(1): 104. doi: 10.15744/23489820.7.104

Received Date: January 27, 2019 Accepted Date: February 25, 2019 Published Date: February 27, 2019

\begin{abstract}
Urinary tract infections (UTIs) are among the most common bacterial infections encountered in adults. Cystitis and lower urinary tract infections are the most common presentations. Most patients are treated successfully; complications are uncommon of which pyelonephritis, recurrent urinary tract infection and sepsis constitute the majority. A rare but serious complication of urinary tract infections is development of a splenic abscess with significant associated mortality.

We describe a 48 year old male patient who presented with urinary tract infection, complicated by multiple splenic abscesses and review all previously described cases.
\end{abstract}

Keywords: Splenic Abscess; Complicated Urinary Tract Infection; Escherichia coli

\section{Introduction}

Splenic abscess is a serious and potentially life threatening complication of UTIs. Although splenic abscesses are a well-known complication of hematogenous infections (mainly endocarditis), and despite the high incidence of UTIs, we rarely encounter them as a complication $[1,2]$.

Splenic abscesses can be easily misdiagnosed as infarcts; therefore, a high degree of suspicion is warranted. Diagnosis is based on imaging studies and treatment is mainly based on prolonged antibiotic administration, drainage, and splenectomy. Early diagnosis is crucial for the proper management and reduction morbidity and mortality rates.

\section{Case Presentation}

This 48 year old patient came to the emergency room because of sudden left lower chest and left upper abdomen stabbing pain, preceded by 10 days of $39-40{ }^{\circ} \mathrm{C}$ fever, shivering, dysuria, headache and weakness. The day before his admission he was diagnosed with UTI by his primary care physician and was started on oral cefuroxime axetil. His previous medical history included hyperlipidemia, hypothyroidism and vitiligo. Three months earlier he was treated for one week with ciprofloxacin for UTI and Escherichia coli was isolated from a urine culture. His medical treatment included simvastatin and eltroxin.

On admission the patient had a $38.5^{\circ} \mathrm{C}$ temperature, the heart rate was $125 / \mathrm{min}$ and his blood pressure was $135 / 80 \mathrm{mmHg}$. His physical examination was otherwise unremarkable except for left upper quadrant abdominal tenderness. Rectal examination showed no tenderness or enlargement of the prostate. Blood tests showed marked leukocytosis $(22.000 / \mu \mathrm{L}$, neutrophils $92 \%)$, an elevated CRP level $(21.2 \mathrm{mg} / \mathrm{dl})$ and a normal PSA level $(0.4 \mathrm{ng} / \mathrm{ml})$. The chest $\mathrm{x}$-ray was normal.

The patient was started on intravenous ceftriaxone $2 \mathrm{~g}$ per day. Blood and urine cultures grew E.coli. Fever and bacteremia continued despite appropriate antibiotic coverage. Botha transthoracic and a transesophageal echocardiogram were done that showed no signs of vegetations or valvular abnormalities. An abdominal ultrasound (US) revealed an enlarged spleen with multiple hypoechoic lesions. CT scan showed an enlarged spleen $(15 \mathrm{~cm})$ with three hypodense lesions. The lesions had no air fluid level and varied in diameter of which the largest was $4 \mathrm{~cm}$ (Figure 1). 


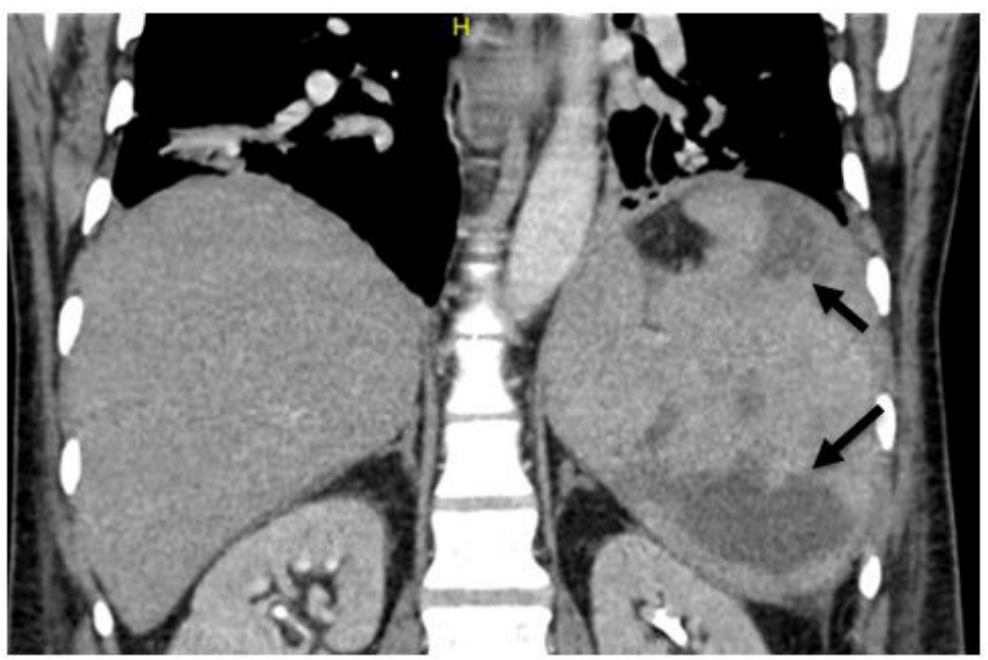

Figure 1: A coronal CT scan of the spleen showing at least two hypodense lesions with no air fluid level(arrows)

Because the patient continued to be febrile the largest of the splenic lesions was aspirated under US guidance. A percutaneous catheter was inserted that drained around $150 \mathrm{ml}$ of pus during 3 days. Culture of the aspirate grew E.coli. The day after draining the abscess the fever subsided. A follow up US confirmed complete evacuation of the abscess; however the other two abscesses were without improvement. Percutaneously draining the remaining abscesses was not an option due to their smaller size and deeper location. During his admission the patient was treated with ceftriaxone alone for 5 days followed by 14 days of ceftriaxone with ciprofloxacin. The patient was discharged on oral ciprofloxacin $750 \mathrm{mg}$ twice daily for another two weeks. During the next 5 months of follow up fever once again relapsed. The US demonstrated that the remaining two abscesses did not decrease in size so he underwent an uneventful laparoscopic splenectomy. The patient completely recovered.

\section{Discussion and Literature Review}

\section{Incidence and Predisposing Factors}

Despite the early description of splenic abscesses by Hippocrates, there are so far less than 1000 cases reported in the literature [3]. With a reported autopsy incidence of $0.14 \%-0.7 \%$ we would expect otherwise $[2,4,5]$. Splenic abscesses are more common among males with a male to female ratio of $2: 1[2,4,5]$. The considerable difference of incidence of splenic abscesses between autopsy reports and cases reported in the literature can be attributed to several factors; most autopsy reports are from the early-mid $20^{\text {th }}$ century, well before the new imaging and treatment techniques; misinterpretation of CT and US findings as infarcts or cysts; under-documentation and reporting of cases.

The most common predisposing factors are metastatic infections, immunodeficiency and abdominal surgery. Percentages are slightly different in different reports [2,4-6]. Endocarditis is by far the most common cause. A thorough MEDLINE/PubMed search revealed only 9 cases of urinary tract infections complicated by splenic abscesses from three reports (Table 1). Chun et al reported in 1980 fourteen cases of splenic abscesses of which three were in association with UTI [5]. The second report was published in 1976 by Chulay et al describing 10 cases of splenic abscesses of which one was associated with UTI [7]. The third report was published in 1998 by Brook et al. The report included 29 cases of splenic abscesses, five of which were in association with UTI [8].

\begin{tabular}{|c|c|c|c|c|c|}
\hline Reference & Year & Age & Gender & Culture & Diagnosed by \\
\hline $\mathbf{5}$ & 1966 & 66 & F & Proteus vulgaris + E.coli & Autopsy \\
\hline $\mathbf{5}$ & 1970 & 61 & M & Proteus & Autopsy \\
\hline $\mathbf{6}$ & 1974 & 79 & F & E.coli & Autopsy \\
\hline $\mathbf{5}$ & 1976 & 47 & F & E.coli & Laparotomy \\
\hline 7 & $1970-1990$ & UA & UA & E.coli & UA \\
\hline 7 & $1970-1990$ & UA & UA & E.coli & UA \\
\hline 7 & $1970-1990$ & UA & UA & E.coli & UA \\
\hline 7 & $1970-1990$ & UA & UA & Proteus & UA \\
\hline 7 & $1970-1990$ & UA & UA & Proteus & UA \\
\hline Current report & 2015 & 48 & M & E.coli & Ultrasound \\
\hline
\end{tabular}

Table 1: Summary of available reports on urinary tract infections complicated with splenic abscess 


\section{Presentation and Diagnosis}

Early diagnosis of splenic abscesses as with other intra-abdominal abscesses is crucial for early intervention and survival [9]. Due to the rarity of the condition the diagnosis is often delayed leading to higher morbidity and mortality [2].

As with our case, the most common findings in patients with splenic abscesses are fever and left upper quadrant abdominal pain/ tenderness [2,4-6]. These findings are not specific or diagnostic of splenic abscesses so a high degree of suspicion is warranted. In patients with an active infectious disease, i.e. endocarditis and UTI, left upper quadrant abdominal pain is not part of the usual presentation and should raise suspicion. Patients with immunodeficiencies or after abdominal surgery presenting with fever and abdominal pain with no clear cause quickly will undergo investigative US or CT scan. If negative and symptoms persist, another look at the spleen may be indicated as small splenic abscesses can be easily missed or interpreted as infarcts. The imaging technique of choice is abdominal CT scan. CT scans usually show a homogeneous low-density area with or without rim enhancement, lucent areas within the spleen containing fluid levels of different densities and intra splenic gas formation [10,11].

The US remains a valuable initial modality due to its accessibility and cost-effectiveness. The US usually shows a hypo echoic or nearly anechoic, ovoid- or round-shaped area in the spleen, with varying internal echogenicity, irregular wall and mild to moderate distal acoustic enhancement [12,13]. Differential diagnosis of splenic abscess in CT and US images include splenic infarct, hematoma, neoplasm and complicated cyst [14].

MRI may be superior to CT in detecting splenic abscesses [15]. However, its availability and cost limit its use to cases where the CT is inconclusive. Fine needle aspirations can also be used to further investigate lesions that are not clear on CT scans.

\section{Management}

Treatment of splenic abscesses is mainly percutaneous drainage and prolonged antibiotic treatment $[8,13]$. Patients having multiple abscesses or showing lack of improvement such as continued fever and persistent bacteremia should be considered for splenectomy.

\section{Conclusion}

We presented a 48 year old male patient suffering from multiple spleen abscesses in association with urinary tract infection. After successfully draining one of the larger abscesses he continued having intermittent fever and elevated inflammatory markers despite prolonged antibiotic treatment. He therefore underwent laparoscopic splenectomy which leads to complete cure.

There are only nine other previously reported cases of splenic abscesses secondary to UTI in the medical literature. The most common predisposing factors for splenic abscesses are endocarditis and immunodeficiency. The most common signs are fever and right upper abdomen pain/tenderness. The diagnostic modality of choice is an abdominal CT scan although US is a valuable initial test. Treatment of splenic abscesses is largely dependent on the size and number of the abscesses. Spleen sparing options should be considered when appropriate including US or CT guided percutaneous drainage and prolonged antibiotic treatment.

\section{Declaration of Conflicting Interests}

None of the authors has any conflicts of interest with the contents.

\section{Funding}

This research received no specific grant from any funding agency in the public, commercial, or not-for-profit sectors.

\section{Ethics and Patient Consent}

\section{Ethics approval}

Our institution does not require ethical approval for reporting individual cases or case series.

\section{Informed consent}

Verbal informed consent was obtained from the patient(s) for their anonymized information to be published in this article.

\section{References}

1. Foxman B (2002) Epidemiology of urinary tract infections: incidence, morbidity, and economic costs. Am J Med 113: 5-13.

2. Nelken N, Ignatius J, Skinner M, Christensen N (1987) Changing clinical spectrum of splenic abscess. A multicenter study and review of the literature. Am J Surg 154: 27-34.

3. Hippocrates (2018) Department Science and Technology. Bibliotheque national de France 8-T: 23-21.

4. Ooi LL, Leong SS (1997) Splenic abscesses from 1987 to 1995. Am J Surg 174: 87-93.

5. Chun CH, Raff MJ, Contreras L, Varghese R, Waterman N, et al. (1980) Splenic abscess. Med (Baltimore) 59: 50-65.

6. Lee CH, Leu HS, Hu TH, Liu JW (2004) Splenic abscess in southern Taiwan. J Microbiol Immunol Infect 37: 39-44. 
7. Chulay JD, Lankerani MR (1976) Splenic abscess. Report of 10 cases and review of the literature. Am J Med 61: 513-22.

8. Brook I, Frazier EH (1998) Microbiology of liver and spleen abscesses. J Med Microbiol 47: 1075-80.

9. Linos DA, Nagorney DM, McIlrath DC (1983) Splenic abscess--the importance of early diagnosis. Mayo Clin Proc 58: 261-4.

10. Tikkakoski T, Siniluoto T, Paivansalo M, Taavistainene M, Leppanen M, et al. (1992) Splenic abscess: Imaging and intervention. Acta Radiologica 33: 561-5.

11. Balthazar EJ, Hilton S, Naidich D, Megibow A, Levine R (1985) CT of splenic and perisplenic abnormalities in septic patients. AJ R Am J Roentgenol 144: 53-6.

12. Rails PW, Quinn MF, Colletti P, Lapin SA, Halls J (1982) Sonography of pyogenic splenic abscess. AJR Am J Roentgenol 138: 523-5.

13. Chou YH, Hsu CC, Tiu CM, Chang T (1992) Splenic abscess: Sono- graphic diagnosis and percutaneous drainage or aspiration. Gastrointest Radiol 17: 262-6.

14. Balcar IB, Seltzer SE, Davis, S, Geller S (1984) CT patterns of splenic infarction: A clinical and experimental study. Radiol 151: 723-9.

15. Elsayes KM, Narra VR, Mukundan G, Lewis JS Jr, Menias CO, et al. (2005) MR imaging of the spleen: spectrum of abnormalities. Radiographics 25: 967-82.

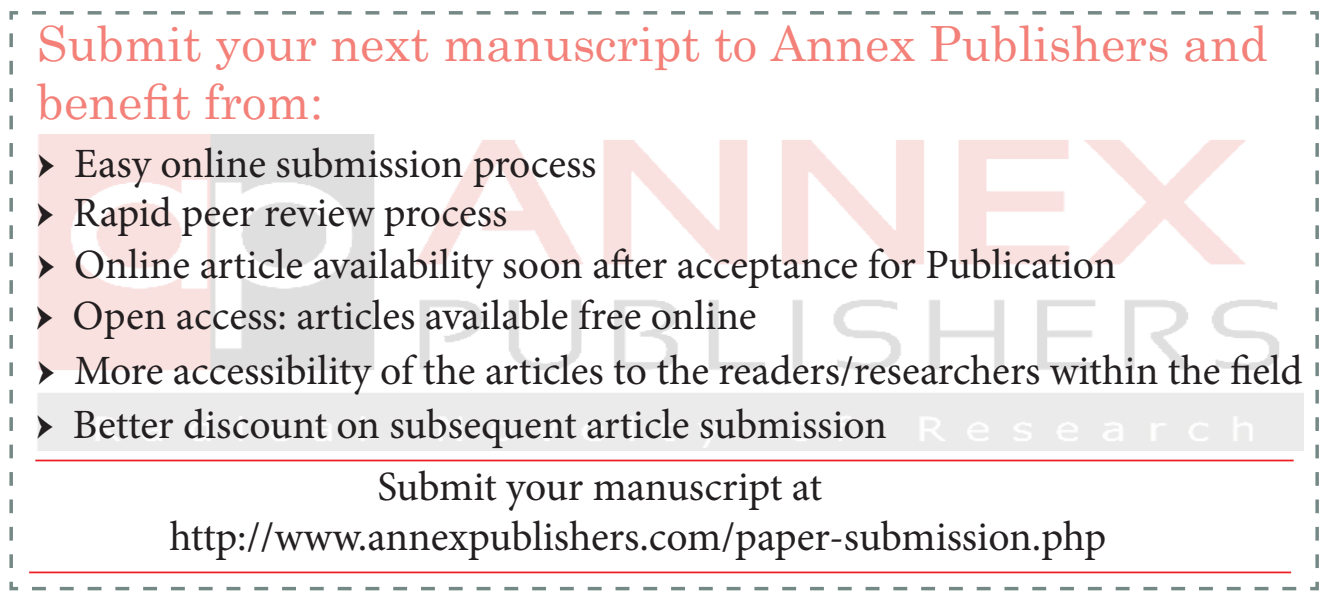

